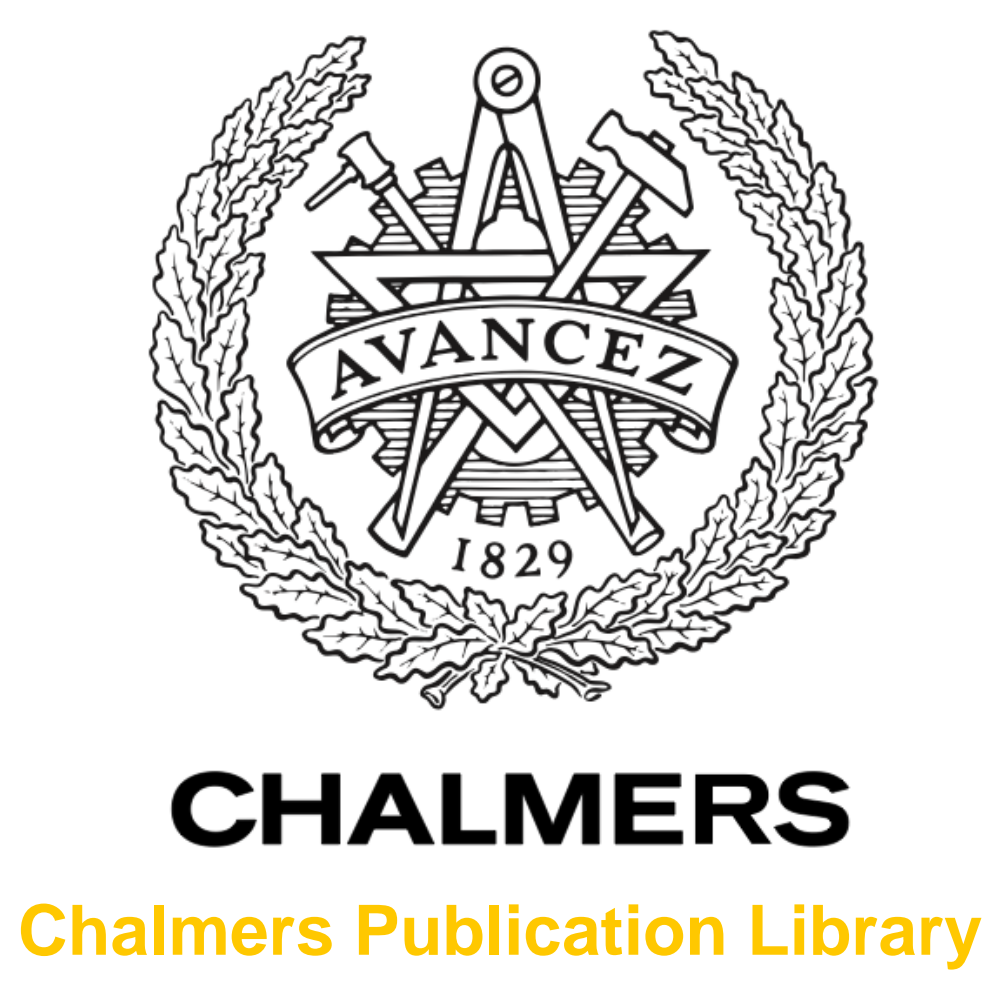

\title{
Rapid determination of bulk microalgal biochemical composition by Fourier- Transform Infrared spectroscopy
}

This document has been downloaded from Chalmers Publication Library (CPL). It is the author's version of a work that was accepted for publication in:

Bioresource Technology (ISSN: 0960-8524)

Citation for the published paper:

Mayers, J. ; Flynn, K. ; Shields, R. (2013) "Rapid determination of bulk microalgal biochemical composition by Fourier-Transform Infrared spectroscopy". Bioresource

Technology, vol. 148 pp. 215-220.

http://dx.doi.org/10.1016/j.biortech.2013.08.133

Downloaded from: http://publications.lib.chalmers.se/publication/241598

Notice: Changes introduced as a result of publishing processes such as copy-editing and formatting may not be reflected in this document. For a definitive version of this work, please refer to the published source. Please note that access to the published version might require a subscription. 


\title{
Rapid determination of bulk microalgal biochemical composition by Fourier-Transform Infrared spectroscopy
}

\author{
Joshua J. Mayers, Kevin J. Flynn*, Robin J. Shields \\ Centre for Sustainable Aquatic Research, Swansea University, Swansea SA2 8PP, UK
}

\section{H I G H L I G H T S}

- A new FTIR-ATR method is proposed for simultaneous measurement of microalgal biochemical content.

- A univariate calibration model was built that allows comparison with conventional techniques.

- High degrees of predictive accuracy were achieved for lipid, protein and carbohydrate.

- Method benefits from small sample requirements, rapid measurement and high reproducibility.

- Technique used to monitor composition of Nannochloropsis sp. culture grown to nitrogen starvation.

\section{A R T I C L E I N F O}

\section{Article history:}

Received 19 July 2013

Received in revised form 20 August 2013

Accepted 23 August 2013

Available online 3 September 2013

\section{Keywords:}

FTIR spectroscopy

Microalgae

Lipids

Process monitoring

Nannochloropsis sp.

\begin{abstract}
A B S T R A C T
Analysis of bulk biochemical composition is a key in fundamental and applied studies of microalgae and is essential to understanding responses to different cultivation scenarios. Traditional biochemical methods for the quantification of lipids, carbohydrates and proteins are often time-consuming, often involve hazardous reagents, require significant amounts of biomass and are highly dependent on practitioner proficiency. This study presents a rapid and non-destructive method, utilising Fourier-Transform Infrared (FTIR) spectroscopy for the simultaneous determination of lipid, protein and carbohydrate content in microalgal biomass. A simple univariate regression was applied to sets of reference microalgal spectra of known composition and recognised IR peak integrals. A robust single-species model was constructed, with coefficients of determination $r^{2}>0.95$, high predictive accuracy and relative errors below $5 \%$. The applicability of this methodology is demonstrated by monitoring the time-resolved changes in biochemical composition of the marine alga Nannochloropsis sp. grown to nitrogen starvation.
\end{abstract}

(c) 2013 Elsevier Ltd. All rights reserved.

\section{Introduction}

It is widely accepted that a greater understanding of the biological processes and abiotic factors influencing the accumulation of desirable metabolites is fundamental in all aspects of biotechnology. That situation is so also for the selection of the appropriate algal strain and cultivation strategies in the rapidly expanding arena of microalgal biotechnology (Griffiths and Harrison, 2009; Greenwell et al., 2010). Numerous studies have documented how environmental factors, culture conditions and growth phase modulate microalgal biochemical composition, with particular interest paid to the lipid fraction as a feedstock for biodiesel (Rodolfi et al., 2009; Breuer et al., 2012). Those wishing to apply metabolic modelling approaches also require detailed biochemical data to ensure high degrees of predictive accuracy (Flynn, 2005). Careful and rapid quantification of the macromolecular groups is therefore of

\footnotetext{
* Corresponding author. Tel.: +44 1792205036.

E-mail address: K.J.Flynn@swansea.ac.uk (K.J. Flynn).
}

high importance for assessing microalgal growth across all scales of production.

The lipid, protein and carbohydrate composition of algal biomass is usually quantified using a diverse range of techniques, typically involving chemical extraction followed by gravimetric determination, spectrophotometry or mass spectroscopy. However, these methods require access to several instrument types, are often inherently time-consuming, and require the use of hazardous reagents. As they are multi-step procedures, they are also particularly prone to human error. The combined effort, coupled with the need for relatively large biomass samples in order to achieve accurate determinations, often results in limited sampling frequency during small-scale trials and batch cultures.

Recently, several techniques have been investigated for more rapid and reproducible measurement of algal biomass composition, including inline spectrophotometry (Solovchenko et al., 2011), Raman spectroscopy (Huang et al., 2010) and nuclear magnetic resonance (NMR) (Davey et al., 2012). Another such technique is Fourier-Transform Infrared (FTIR) spectroscopy, which 
represents a rapid, direct and non-destructive method for the simultaneous measurement of bulk biochemical composition. This work explores the use of FTIR spectroscopy as applied to microalgae.

Infrared (IR) spectroscopy exploits the fact that different molecules absorb specific frequencies (wavenumber) of infrared radiation $\left(14,000-10 \mathrm{~cm}^{-1}\right)$. Each chemical bond in a molecule vibrates at specific frequencies; when the vibrational frequency of the bond matches that of the infrared radiation, absorption occurs (Movasaghi et al., 2008). Analysis of the composition, shape and intensity of absorption at specific wavenumbers reveals information about functional groups and molecular structure that can then be used for identification.

Absorbance of IR at a given wavenumber is proportional to the concentration of the corresponding bond or molecule, leading to a general pattern of behaviour that follows Beer's Law.

$A_{v}=\varepsilon_{v} b c$

Where $A_{v}$ is the absorbance at wavenumber $v, \varepsilon_{v}$ is the molar absorption coefficient of the material, $b$ is the pathlength of the sample, and $c$ is the concentration of the material in that sample. This allows not just qualitative observation, but also the ability to quantify the presence of functional groups, molecules or whole biochemical fractions.

Different macromolecular groups (lipids, carbohydrates and proteins) contain characteristic functional groups and chemical bonds that allow identification and quantification based upon absorbance. For samples containing a complex mixture of biochemical groups such as those found in cellular material, spectra are accordingly highly complex. Two regions in the mid-infrared range $\left(4000-400 \mathrm{~cm}^{-1}\right)$ between $3000-2800 \mathrm{~cm}^{-1}$ and $1800-$ $900 \mathrm{~cm}^{-1}$ (Movasaghi et al., 2008), consisting of numerous overlapping peaks, account for the majority of biochemical information. With appropriate software and configuration, FTIR has found applications in process engineering and in biotechnological settings for process monitoring, quality assurance and validation purposes (Nicolai et al., 2007; Sills and Gossett, 2012) for several biomass types (Pistorius et al., 2009).

In relation to microalgae, FTIR has been used to analyse a number of parameters, including growth prediction (Jebsen et al., 2012), changes in elemental carbon allocation (Palmucci et al., 2011) and physiological responses to nutrient stress (Murdock and Wetzel, 2009; Dean et al., 2010; Wagner et al., 2010). Several studies have examined the relative content of microalgal macromolecules by comparing the ratio of particular absorption bands (Giordano et al., 2001; Stehfest et al., 2005; Dean et al., 2010).

Two methods that sought to measure the absolute quantities of different biochemical groups in microalgae have been developed. Pistorius et al. (2009) developed a simple linear model for determining the approximate biochemical composition for microbial samples (bacteria, yeast, microalgae) using peak integration at regions corresponding to particular biochemical groups and generated standard curves using exogenous reference standards. Wagner et al. (2010) took a very similar approach but used the spectra of reference standards to reconstruct sample cell spectra and used multivariate PLS regression to infer the quantities of biochemical groups in the green alga Chlamydomonas reinhardtii. Methodologies and equations from these studies have subsequently been used in other publications (Feng et al., 2012; Jungandreas et al., 2012). In another study, acceptable accuracy in determining lipid content was achieved using a multivariate PLS model, where the spectra of two exogenous lipid standards were calibrated against algal biomass spiked at different levels with the same lipids (Laurens and Wolfrum, 2011).

The present study proposes a simple linear regression method to accurately quantify microalgal biochemical composition. The alga used as an example in this study is the marine species Nannochloropsis sp., which is already a commonly cultivated aquaculture species and a promising candidate for biofuel and nutraceutical production (Chiu et al., 2009; Nobre et al., 2013). This is the first time an FTIR spectroscopic method is utilized for the simultaneous determination of microalgal biomass, utilising regression models built from reference microalgal spectra and biochemical composition data gathered using conventional analytical techniques, allowing direct comparisons with data in the literature. The chosen approach also benefits from using an attenuated total reflectance (ATR) sampling technique, further increasing the speed, ease and reproducibility of analysis. The aim is to allow for closer monitoring of microalgae production systems and to achieve more detailed and efficient optimization of process design and biomass production strategies.

\section{Methods}

\subsection{Culture conditions and sample preparation}

The green marine microalga Nannochloropsis sp. (CCAP 211/78) was cultivated in $10 \mathrm{~L}$ tubular airlift bioreactors (acrylic plastic, $0.1 \mathrm{~m}$ diameter/light path, $1.2 \mathrm{~m}$ height) in batch cultures. Reactors were maintained at $19-21^{\circ} \mathrm{C}$, aerated with filtered $(0.2 \mu \mathrm{m})$ ambient air $\left(0.039 \% \mathrm{CO}_{2}\right)$ at a rate of $0.1 \mathrm{~L}^{-1}(\mathrm{v} / \mathrm{v})$ into the base of the tube through a $1 \mathrm{~mm}$ plastic capillary tube. Bioreactors were illuminated at the reactor surface with approximately $60 \mu \mathrm{mol}$ photons $\mathrm{m}^{-2} \mathrm{~s}^{-1}$ using cool white fluorescent tubes perpendicular to the bioreactors, with a light/dark cycle of $18 / 6 \mathrm{~h}$.

Cultures were grown either on modified Walnes media (Andersen, 2005) or on anaerobic digestate effluent (ADE) media with initial nitrogen concentrations of $5,10,20$ or $60 \mathrm{mg} \mathrm{L}^{-1}$, and nitrogen and phosphorus molar ratios of $16: 1,32: 1$ and $64: 1$. Inorganic nitrogen and phosphate was supplied as sodium nitrate and potassium dihydrogen phosphate, respectively. The main nitrogen source in the ADE was ammonium, but was supplemented with the inorganic nutrient sources as above to make up to the desired nutrient concentration and N:P ratio. Samples were collected every other day throughout the growth cycle until cultures reached stationary phase. The use of samples grown under a variety of nutrient regimes and harvested at different time points, yielded biomass samples of various biochemical composition.

\subsection{Analytical methods}

\subsubsection{Culture density and biomass concentration}

Total cellular volume was recorded using a Coulter counter (C3 Beckman Coulter GmBH, Drefield, Germany) and equated to dry weight using an appropriate calibration curve. Dry weight was determined by filtering a known volume of culture onto precombusted GF/F Whatmann filters. Filters were washed with ammonium formate $(0.5 \mathrm{M})$ to remove salts, then dried for at least 18 h at $70^{\circ} \mathrm{C}$.

\subsubsection{Elemental carbon and nitrogen}

A known volume of culture suspension was filtered onto a precombusted $550^{\circ} \mathrm{C}, 20 \mathrm{~min}$ ) $13 \mathrm{~mm} \mathrm{~A} / \mathrm{E}$ glass fibre filter (Pall) at room temperature, dried at $60^{\circ} \mathrm{C}$ for $24 \mathrm{~h}$, wrapped in tin discs (Exeter Analytical, UK) and analysed for total nitrogen and total carbon using a SerCon GSL elemental analyser $\left(1000{ }^{\circ} \mathrm{C}\right.$ combustion temperature) interfaced with 20-20 Isotope Ratio Mass Spectrometer (PDZ-Europa, UK). Operation follows standard methods published by the instrument manufacturers, with the exception of oxidation column being packed solely with chrome oxide, rather a mix of chrome oxide and copper oxide, which prevents 
premature column failure when analysing samples of marine origin. Measurements were calibrated using isoleucine standards as it has a C:N ratio is of the appropriate value for the unknowns and were placed every 10-12 samples. All measurements were made in duplicate.

\subsubsection{Biochemical composition analysis}

Biomass samples for further analysis were harvested by centrifugation (8000g, $4{ }^{\circ} \mathrm{C}$, JA-2, Beckman, Germany), resuspended and washed twice in deionised water and frozen at $-30^{\circ} \mathrm{C}$ overnight before freeze-drying for $24 \mathrm{~h}$ and being finely ground using a pestle and mortar. Samples were then stored at $-80^{\circ} \mathrm{C}$ prior to analysis. Total lipid content was determined gravimetrically by a modified method according to Folch et al. (1957), whereby $10-15 \mathrm{mg}$ of lyophilised biomass is resuspended in $6 \mathrm{~mL}$ of chloroform methanol $(2: 1, v / v)$ in $10 \mathrm{~mL}$ Teflon lined tubes (Oakridge, Nalgene) and sonicated at $4{ }^{\circ} \mathrm{C}$ for $45 \mathrm{~min}$. This is then held for $24 \mathrm{~h}$ at $4{ }^{\circ} \mathrm{C}$, followed by sonicatication for a further $45 \mathrm{~min}$. To separate phases, $1.5 \mathrm{~mL}$ of $0.9 \%(\mathrm{w} / \mathrm{w}) \mathrm{NaCl}$ is then added to each tube and centrifuged for $8 \mathrm{~min}$ at $2000 \mathrm{rpm}$ (MSE Harrier centrifuge, swing-out $18 / 80 \mathrm{R}$ rotor). The lipid-containing (lower) phase is then recovered and the extraction process repeated a second time by addition of a further $2 \mathrm{~mL}$ of chloroform. The extracts were combined and solvent removed under nitrogen and the lipid then weighed.

Total carbohydrate content was determined using the phenol/ sulphuric acid methodology on $5 \mathrm{mg}$ of lyophilised biomass. Quantification was performed using a glucose standard curve measured at $485 \mathrm{~nm}$ (Dubois et al., 1956).

Protein content was determined by multiplying the nitrogen content of dried biomass, as determined from the elemental analysis, by a factor of 6.28 (Safi et al., 2013). This approach is analogous to that based upon Kjeldahl digestion (Safi et al., 2013).

All biochemical measurements were made in triplicate, except protein, which was performed in duplicate.

\subsection{FTIR spectra collection and pre-processing}

FTIR attenuated total reflectance (ATR) spectra were collected on a PerkinElmer Spectrum Two instrument equipped with a diamond crystal iATR reflectance cell with a DTGS detector scanning over the wavenumber range of $4000-450 \mathrm{~cm}^{-1}$ at a resolution of $4 \mathrm{~cm}^{-1}$. Approximately 3-5 $\mathrm{mg}$ of finely powdered freeze-dried biomass prepared in the same manner as for conventional biochemical analysis (described in Section 2.2.2) was applied to the surface of the crystal and then pressed onto the crystal head. Three replicates (each consisting of an average of 8 scans) for each sample were taken and the results averaged. Background correction scans of ambient air were made prior to each sample scan. Scans were recorded using the spectroscopic software Spectrum (version 10. PerkinElmer, Germany). Spectra were background corrected for ambient air. Ethanol was used to clean the diamond ATR between samples.

\subsection{Quantification method and statistical analysis}

The infrared spectra for the 30 microalgal reference samples were imported into the spectral manipulation package Spectrum Quant (PerkinElmer, Germany). Absorption bands were selected by plotting the integrated area against the percentage of biochemical group in the standard (\% DW). Tangential baselines were selected between two points, for which the area under the absorption band was measured. Least squares polynomial regression analysis yielded the coefficients of the straight lines through the data points for the individual constituents, providing calibrations of peak area against biochemical content. Spectrum Quant (PerkinElmer, Germany) was used for analysis of all infrared spectra and model calibration. Microsoft Excel 2007 was used for processing of growth data.
The predictive performance of the spectral analysis versus data derived from biochemical models was evaluated using various approaches; the coefficient of determination $r^{2}$, the slope (the coefficient of regression of predicted against actual values), the root mean standard of error performance (RMSEP), the root mean square error of cross-validation (RMSECV) generated using a leave-one-out cross-validation ( $\mathrm{LOO}-\mathrm{CV})$, residual predictive deviation (RPD) and finally the relative error (Nicolai et al., 2007). $r^{2}$ represents the difference between true and calculated values of the respective statistical model and the RMSEP and RMSECV serve as standards to assess the quality of the predictive capacity of the model and give the approximate standard error between true and calculated values. RPD is a qualitative measure for the assessment of the validation results (Nicolai et al., 2007).

The cross-validation model offers another approach to estimate the predictive capacity of the model. A LOO-CV is performed by sequentially removing each of the $n$ calibration samples from the model; the resulting model then independently estimates the value of the excluded sample (Brereton, 2009). This process is repeated for all calibration samples and the values averaged. The following equations were used:

$$
\begin{aligned}
& \text { RMSEP }=\sqrt{\frac{\sum_{i=1}^{n}\left(\hat{y}-y_{i}\right)^{2}}{n}} \\
& \text { RMSECV }=\sqrt{\frac{\sum_{i=1}^{n}\left(\hat{y}-y_{i}\right)^{2}}{n-1}} \\
& \operatorname{RPD}=\frac{\text { SD }}{\operatorname{RMSECV}}
\end{aligned}
$$

Relative error $=\frac{\text { RMSECV }}{\text { mean }} \times 100$

$y_{i}$ is the value derived from conventional analytical methodology for the $i^{\text {th }}$ sample; $\hat{y}$ is the predicted value from the model for the same sample; $n$ is the number of samples in each set; SD is the standard deviation in each set.

\section{Results and discussion}

\subsection{Microalgal biochemical composition}

The microalgal samples used in this study were grown under a variety of nutrient conditions and sampled at different time points in growth curves, yielding samples which were either nitrogen or phosphorus deplete or both concurrently; providing a large range of values for lipids, proteins and carbohydrates. In Nannochloropsis sp., the lipid content varied more significantly (21.3-59.2\% DW) than the protein and carbohydrate content (15.6-32.5\% DW and 15.7-30.9\% DW, respectively). Mean values were 43.0\%, $22.2 \%$ and $24.7 \%$ for lipid, protein and carbohydrate, respectively. The residual fraction of measured biomass, typically referred to as ash content, varied between 5 and 10\% DW and is composed of minerals, metals and salts incombustible at $550{ }^{\circ} \mathrm{C}$. The range of values found here are typical for many lipid accumulating microalgae (Laurens et al., 2012; Chiu et al., 2009; Gu et al., 2012; Breuer et al., 2012).

\subsection{Microalgal FTIR spectra}

The general shape of the spectra collected for Nannochloropsis sp. showed different peak intensities at particular wavenumbers depending on the growth conditions (and hence biochemical 
Table 1

Main absorption bands of microalgae in the IR.

\begin{tabular}{|c|c|c|}
\hline $\begin{array}{l}\text { Wavenumber } \\
\left(\mathrm{cm}^{-1}\right)\end{array}$ & $\begin{array}{l}\text { Band } \\
\text { assignment }\end{array}$ & Functional groups \\
\hline $3400-3200$ & ${ }_{v} \mathrm{O}-\mathrm{H} /{ }_{V} \mathrm{~N}-\mathrm{H}$ & Water, protein \\
\hline 2960 & Vas $\mathrm{CH}_{3}$ & $\mathrm{CH}_{3}$ methyl group \\
\hline 2930 & Vas $\mathrm{CH}_{2}$ & $\mathrm{CH}_{2}$ methylene group \\
\hline 2850 & ${ }_{v} \mathrm{CH}_{2},{ }_{v} \mathrm{CH}_{3}$ & $\mathrm{CH}_{2}$ and $\mathrm{CH}_{3}$ methyl \& methylene groups \\
\hline 1745 & ${ }_{v} \mathrm{C}=\mathrm{O}$ & Ester of lipids and fatty acids \\
\hline 1655 & ${ }_{V} \mathrm{C}=\mathrm{O}$ & Protein (Amide I) \\
\hline 1545 & ${ }_{\delta} \mathrm{N}-\mathrm{H},{ }_{v} \mathrm{C}-\mathrm{N}$ & Protein (Amide II) \\
\hline 1455 & ${ }_{\delta a s} \mathrm{CH}_{2},{ }_{\delta a s} \mathrm{CH}_{3}$ & $\mathrm{CH}_{2}$ and $\mathrm{CH}_{3}$ methyl \& methylene groups \\
\hline 1390 & $\begin{array}{l}{ }_{\delta} \mathrm{CH}_{2} \\
\mathrm{CH}_{3} /_{\delta} \mathrm{C}-\mathrm{O}\end{array}$ & $\begin{array}{l}\mathrm{CH}_{2} \text { and } \mathrm{CH}_{3} \text { of proteins/carboxylic } \\
\text { groups }\end{array}$ \\
\hline 1240 & Vas $\mathrm{P}=\mathrm{O}$ & $\begin{array}{l}\text { Phosphodiester of nucleic acids and } \\
\text { phospholipids }\end{array}$ \\
\hline $1200-900$ & ${ }_{v} \mathrm{C}-\mathrm{O}-\mathrm{C}$ & Polysaccharides/siloxane \\
\hline 1075 and 950 & ${ }_{v} \mathrm{Si}-\mathrm{O}$ & Siloxane, silicate frustules \\
\hline $980-940$ & $\mathrm{P}-\mathrm{O}-\mathrm{P}$ & Polyphosphate \\
\hline
\end{tabular}

$V=$ symmetric stretching, vas $=$ asymmetrical stretching, ${ }_{\delta}=$ symmetric deformation (bend), sas $_{\text {= }}$ asymmetric deformation (bend).

Band assignments are taken from references Giordano et al., 2001; Movasaghi et al., 2008; Murdock and Wetzel, 2009.

content). The majority of variation was seen in the spectral range $1700-850 \mathrm{~cm}^{-1}$, with all macromolecular groups displaying the expected distinctive bands (Giordano et al., 2001). The spectral region between 3000 and $2500 \mathrm{~cm}^{-1}$ also showed variation between samples, but changes in peak intensity were not found to correlate highly to changes in biochemical composition. Strong absorption bands at approximately 1050, 1150, 1540, 1650 and $1740 \mathrm{~cm}^{-1}$ dominate spectra for Nannochloropsis sp. (see Supplementary data Fig. 1). These correspond to the $\mathrm{P}=\mathrm{O}$ bond of nucleic acids, the vibration of $\mathrm{C}-\mathrm{O}$ bonds of polysaccharides, the $\mathrm{N}-\mathrm{H}$ of Amide I and $\mathrm{C}=\mathrm{O}$ of Amide II belonging to protein groups (Giordano et al., 2001) and the stretching $C=0$ bonds of esters of lipids and fatty acids, respectively. A more detailed assessment of band assignments for algal functional groups is given in Table 1 .

\subsection{Determination of biomass composition by FTIR}

Two regions are commonly used for the assessment of lipid content by FTIR in the literature (Pistorius et al., 2009; Laurens and Wolfrum, 2011), the methyl and methylene groups at 2800$3000 \mathrm{~cm}^{-1}$ and the vibrational stretching of ester bonds at $1740 \mathrm{~cm}^{-1}$. The latter had a higher linear correlation to lipid content than the methyl and methylene stretching bands in the current study. Methyl group-containing neutral amino acids and carbohydrates have previously been found to contribute to vibrations in this region. Additionally, the large variation in the overlapping $\mathrm{O}-\mathrm{H}$ bond seen at $3100-2800 \mathrm{~cm}^{-1}$ could aid in delineation of the relationship between absorption peak area and lipid content. The vibrational stretching of the $\mathrm{C}=\mathrm{O}$ bond at $1740 \mathrm{~cm}^{-1}$ is considered to be solely due to the ester bonds of fatty acids (Movasaghi et al., 2008) which may explain the results found in this study (Table 2).

The amide II band is widely considered to be the most reliable region for quantification of protein content (Pistorius et al., 2009), and in the present study was found to show a greater linear relationship to protein content in biomass samples than the amide I band (Table 2). Carbohydrates represent a complex group in algal biomass, consisting of a range of long, branched structural polysaccharides and frequently large reserves of storage carbohydrates such as starch and glycogen (González-Fernández and Ballesteros, 2012). Mid-IR spectra of glucose and starch hydrolysate have shown absorption patterns consisting of complex overlapping bands between 1200 and $1000 \mathrm{~cm}^{-1}$ (Pistorius et al., 2009; Wagner et al., 2010). This same region has also been found to contain bands relating to $-\mathrm{PO}_{2}-$ symmetric stretching of phospholipids and DNA, as well as the contribution of the $\mathrm{Si}-\mathrm{O}$ stretching vibration at $1075 \mathrm{~cm}^{-1}$ making resolution of the carbohydrate $\mathrm{C}-\mathrm{O}$ stretching vibrational bond inherently difficult. However, the pronounced characteristic peak at $1050 \mathrm{~cm}^{-1}$ has been found to correlate well to carbohydrate content in biomass samples (Pistorius et al., 2009; Wagner et al., 2010) and was also found to exhibit a good linear correlation to measured carbohydrate content in this study (Table 2).

Cross-validation of the lipid, protein and carbohydrate models show high degrees of statistical accuracy with RMSECV values of approximately $1 \%$ and the coefficient of regression between the actual and predicted values (slope) being $0.989,0.976$ and 0.930 , respectively (Fig. 1). The RPD values were all high (>3), indicating good predictive accuracy (Nicolai et al., 2007). The relative error values of lipids, carbohydrates and proteins were all $<5 \%$, indicating that the regression relationships are reliable.

\subsection{Application of developed models for high resolution monitoring of Nannochloropsis sp. batch culture}

Fig. 2 shows the growth curve and changes in carbon:nitrogen molar ratio of Nannochloropsis sp. during a 10-day growth trial in a tubular bioreactor system under 18/6 light dark cycles, while Fig. 3 displays the corresponding changes in biochemical composition.

The initial extracellular nitrogen content (supplied in the form of $\left.\mathrm{NO}_{3}\right)$ was $10 \mathrm{mg} \mathrm{L}^{-1}(16: 1 \mathrm{~N}: \mathrm{P})$ and was depleted by day 6 . The culture inoculum was partially nitrogen depleted at the beginning of the experiment, shown by slightly elevated $\mathrm{C}: \mathrm{N}$ ratio, high lipid content and low protein content. This was found to change over the next 2-4 days as abundant extracellular nitrogen allowed protein synthesis to recommence, and the lipid content and $\mathrm{C}: \mathrm{N}$ ratio to subsequently decreased. After exhaustion of the extracellular nitrogen source (day 6) the C:N ratio increased (Fig 2), with the protein and lipid fractions showing a characteristic reversal in concentration (Fig. 3). The lipid and carbohydrate fractions

Table 2

Performance of statistical models for prediction of biochemical content in Nannochloropsis sp. using standards of known composition.

\begin{tabular}{|c|c|c|c|c|c|c|}
\hline Component & $r^{2 a}$ & Slope $^{b}$ & RMSEP $^{\mathrm{c}}$ & RMSECV $^{\mathrm{d}}$ & Relative error $^{\mathrm{e}}(\%)$ & $\mathrm{RPD}^{\mathrm{f}}$ \\
\hline Lipid & 0.995 & 0.989 & 1.163 & 1.278 & 2.97 & 8.95 \\
\hline Protein & 0.969 & 0.976 & 1.035 & 1.013 & 4.56 & 5.29 \\
\hline Carbohydrate & 0.965 & 0.930 & 1.254 & 1.223 & 4.94 & 3.44 \\
\hline
\end{tabular}

$n=30$.

a Coefficient of determination.

b Coefficient of regression of predicted against actual values.

c Root mean-square error of performance.

d Root mean-square error of cross-validation.

e Percentage of RMSEP to mean.

${ }^{\mathrm{f}}$ Residual prediction deviation. 

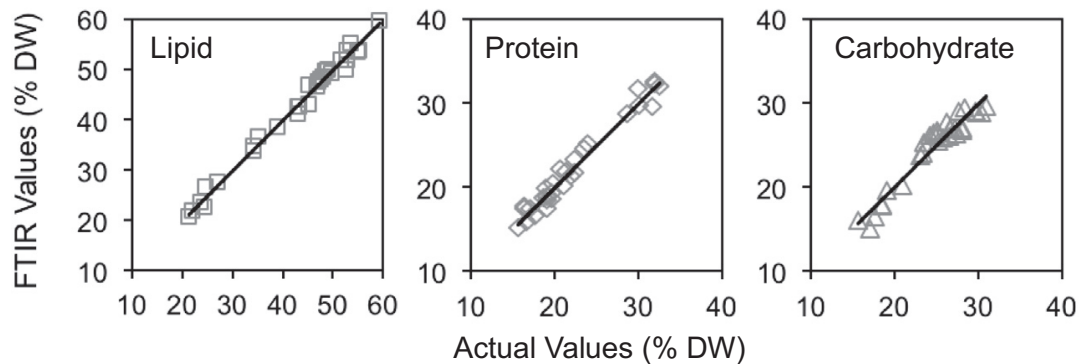

Fig. 1. Plots of FTIR predicted versus actual biochemical values of each biochemical component (\% DW) in Nannochloropsis sp.

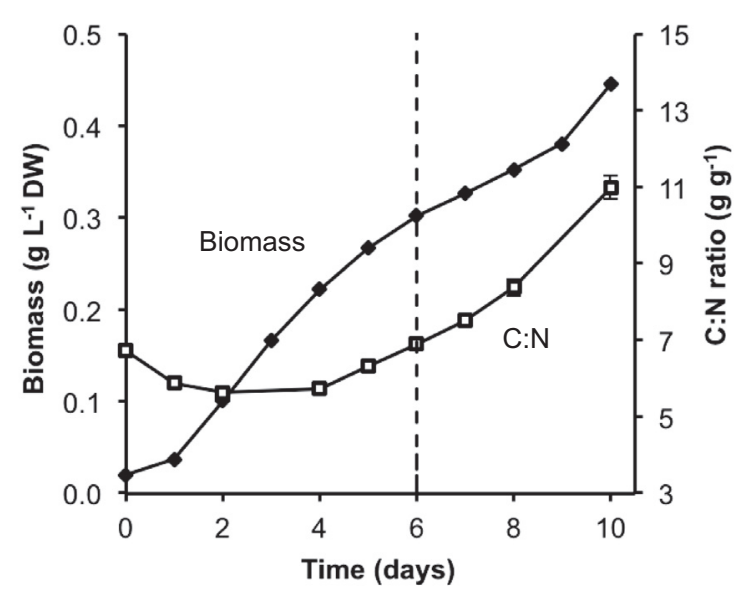

Fig. 2. Biomass concentration and carbon to nitrogen mass ratio $(\mathrm{mg} / \mathrm{mg})$ over a 10 day batch growth on inorganic media ( $: \mathrm{N}, n=2$, error bars $=1 \mathrm{SD})$. Dashed line indicates full nitrogen depletion.

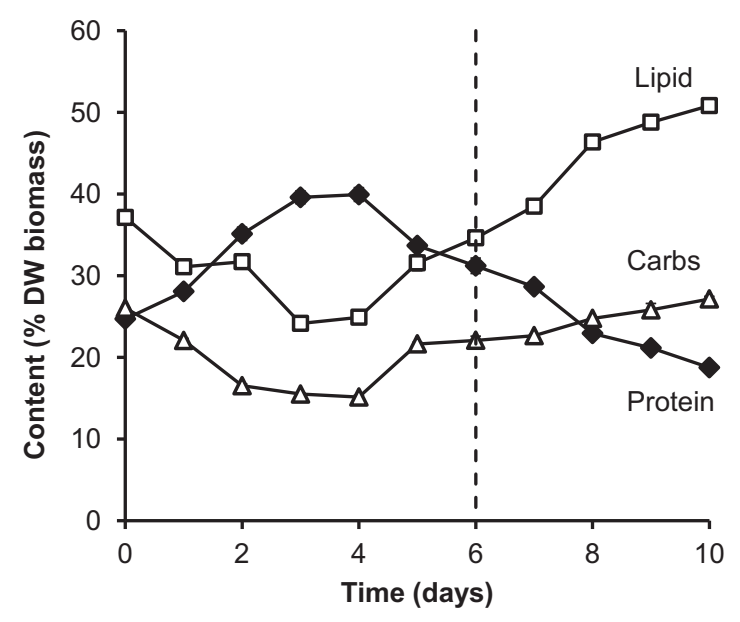

Fig. 3. Time resolved changes in protein, lipid carbohydrate content (\% DW) between day 0 and 10 . Values determined by FTIR ( $n=3$, error bars $=1$ SD). Dashed line indicates full nitrogen depletion.

increased by $210 \%$ and $180 \%$ from the minimum values, respectively, while the protein decreased by $47 \%$ amount over the same period. In accordance with the literature, in this study Nannochloropsis sp. accumulated lipids rather than carbohydrates under nitrogen stress (Chiu et al., 2009; Gu et al., 2012). Biomass accumulation continued up to day 10 with little increase in cell density (not shown), indicating that metabolism had switched to accumulation of carbon storage compounds rather than division. These results indicate that the infrared methods developed in this study can rapidly and simultaneously monitor and quantify the large changes in microalgal biochemical composition during typical growth experiments.

\subsection{Method considerations and perspectives}

Compared to traditional methods, FTIR required as little as 3$5 \mathrm{mg}$ of dry biomass to obtain suitable spectra and that biomass is often recoverable. This compares with the need for between 50 and $100 \mathrm{mg}$ for wet-chemical methods (Laurens et al., 2012). FTIR thus allows a greater sampling frequency than previously achievable from small-scale culture system in comparison with numeric values generated using standard methodologies, while also offering a large time saving and much greater simplicity.

Many of the traditional methods used for monitoring bulk biochemical composition in microalgae involve multi-step procedures, often-making use of chemical extractions and spectrophotometric techniques. Subsequently these techniques are inherently time consuming, resource dependent and often require the use of hazardous reagents, but also suffer from wellknown sources of error (Dubois et al., 1956; Doan and Obbard, 2011; Laurens et al., 2012; Safi et al., 2013; Templeton et al., 2012).

Despite known problems, these methods are still in routine use and any new methods should be comparable to existing methods, as is the case with the proposed technique. The timesaving offered by the determination of all three components simultaneously using a single instrument for FTIR spectroscopy is considerable. Sample preparation is simple (the same as used as a pre-requisite for traditional methods) and spectra acquisition is rapid, with measurement of a sample in triplicate completed in less than $15 \mathrm{~min}$ and with a high degree of accuracy. Additionally, the utilisation of the attenuated-total reflectance (ATR) sampling technique decreases the errors and spectral variations often experienced using other spectroscopic sampling techniques. Other studies have used FTIR spectroscopy in a transmission mode of sampling, whereby samples are deposited on slides or plates (Wagner et al., 2010). This also benefits from having a low sample requirement and being high in throughput, but factors such as sample thickness, homogeneity and sample preparation can contribute markedly to low spectra quality and poor reproducibility. As such these methods often require complex baseline correction algorithms and spectral pretreatments to obtain accurate measurements. The method in the present study benefits from faster sampling and improved sample-to-sample and user-to-user reproducibility.

The use of samples of great biochemical diversity and concentration range is important in order to construct models for the prediction of various compositions. Each biochemical group can exhibit significant changes not just in concentration but also in composition. Lipid composition is often highly diverse, with significant changes in chain length, saturation and the presence of 
hydrophilic groups observed under different culture conditions (Guschina and Harwood, 2006). However, the determination of protein can be assumed to be less affected by changes in aminoacid composition as the amide bands originate primarily from their secondary structure between peptide groups (Wagner et al., 2010). The use of actual biochemical data, derived from a complex matrix such as whole cells, to construct models rather than from standards allows variation within these pools to be considered by including samples grown under a range of conditions in the calibration. This is also a much better demonstration of how robust a technique it is, as it represents a wide-range of sample states and, more importantly, means that predicted concentrations can be directly compared with those generated by conventional biochemical techniques.

\section{Conclusion}

The proposed FTIR technique offers a rapid, accurate and highly reproducible method that has been demonstrating for quantification of large changes in biochemical composition during typical growth experiments. Results are also directly comparable with those from conventional methods, but using relatively small sample volumes. The use of this approach should enable the more ready generation of the types of data required for the optimisation of cultivation strategies and configuration of mathematic models describing the growth of microalgae. The next challenge is thus to speed up sample preparation, to enable the approach to become closer to real-time in capability.

\section{Acknowledgements}

The authors would like to gratefully thank Naomi Ginnever, Kelly Palmer, Aaron Davies, Alla Silkina, Shaun Richardson and all the CSAR technical team for their help, time and support. The work of Joshua Mayers is funded by the European Structural Funds via a Knowledge Economy Skills Scholarship. The authors would also like to acknowledge the financial support of the INTEREG IVB Northwest Europe programme, via the EnAlgae project.

\section{Appendix A. Supplementary data}

Supplementary data associated with this article can be found, in the online version, at http://dx.doi.org/10.1016/j.biortech.2013. 08.133 .

\section{References}

Andersen, R.A., 2005. Algal Culturing Techniques. Elsevier Academic Press, USA. Brereton, R., 2009. Chemometrics for Pattern Recognition. John Wiley \& Sons, UK. Breuer, G., Lamers, P.P., Martens, D.E., Draaisma, R.B., Wijffels, R.H., 2012. The impact of nitrogen starvation on the dynamics of triacylglycerol accumulation in nine microalgae strains. Bioresour. Technol. 124, 217-226.

Chiu, S.-Y., Kao, C.-Y., Tsai, M.-T., Ong, S.-C., Chen, C.-H., Lin, C.-S., 2009. Lipid accumulation and $\mathrm{CO}_{2}$ utilisation of Nannochloropsis oculata in response to $\mathrm{CO}_{2}$ aeration. Bioresour. Technol. 100, 833-838.

Davey, P.T., Hiscox, W.C., Lucker, B.F., O’Fallon, J.V., Chen, S., Helms, G.L., 2012. Rapid triacylglyceride detection and quantification in live micro-algal cultures via liquid state ${ }^{1} \mathrm{H}$ NMR. Algal Res. 1, 166-175.

Dean, A., Sigee, D., Estra, B., Pittman, J., 2010. Using FT-IR spectroscopy for rapid determination of lipid accumulation in response to nitrogen limitation in freshwater microalgae. Bioresour. Technol. 101, 4499-4507.

Dubois, M., Gilles, K.A., Hamilton, J.K., Rebers, P.A., Smith, F., 1956. Colorimetric method for the determination of sugars and related substances. Anal. Chem. 28, $350-356$.

Doan, T.-T.Y., Obbard, J.P., 2011. Improved Nile red staining of Nannochloropsis sp. J. Appl. Phycol. 23, 895-901.

Feng, G.D., Zhang, F., Cheng, L.H.., Xu, X.H., Zhang, L., Chen, H.L., 2012. Evaluation of FT-IR and Nile red methods for microalgal lipid characterisation and biomass composition determination. Bioresour. Technol. 128, 107-112.
Folch, J., Lees, M., Sloane-Stanley, G.H., 1957. A simple method for the isolation and purification of total lipids from animal tissues. J. Biol. Chem. 226, 497-509.

Flynn, K.J., 2005. Castles built on sand: dsyfunctionality in plankton models and the inadequacy of dialogue between biologists and modellers. J. Plank. Res. 27, 1205-1210.

Giordano, M., Kansiz, M., Heraud, P., Beardall, J., Wood, B., McNaughton, D., 2001. Fourier Transform infrared spectroscopy as a novel tool to investigate changes in intracellular macromolecular pools in the marine microalgae Chaetoceros muelleri (Bacillariophyceae). J. Phycol. 37, 271-279.

González-Fernández, C., Ballesteros, M., 2012. Linking microalgae and cyanobacteria culture conditions and key-enzymes to carbohydrate accumulation. Biotechnol. Adv. 30, 1655-1661.

Greenwell, H.C., Laurens, L.M.L., Shields, R.J., Lovitt, R.W., Flynn, K.J., 2010. Placing microalgae on the biofuels priority list: a review of the technological challenges. J. R. Soc. Interface 7, 703-726.

Griffiths, M.J., Harrison, S.T.L., 2009. Lipid productivity as a key characteristic for choosing algal species for biodiesel production. J. Appl. Phycol. 29, 493-507.

Gu, N., Lin, Q., Li, G., Tan, Y., Huang, L., Lin, J., 2012. Effect of salinity on growth, biochemical composition, and lipid productivity of Nannochloropsis oculata CS 179. Eng. Life Sci. 12, 1-7.

Guschina, I.A., Harwood, J.L., 2006. Lipids and lipid metabolism in Eukaryotic algae. Prog. Lipid Res. 45, 160-168.

Huang, Y.Y., Beal, C.M., Cai, W.W., Ruoff, R.S., Terentjev, E.M., 2010. Micro-Raman spectroscopy of algae: Composition analysis and fluorescence background behaviour. Biotechnol. Bioenerg. 105, 889-898.

Jebsen, C., Norici, A., Wagner, H., Palmucci, M., Giordano, M., Wilhelm, C., 2012. FTIR spectra of algal species can be used as physiological fingerprints to assess their actual growth potential. Physiol. Plant 146, 427-438.

Jungandreas, A., Wagner, H., Wilhelm, C., 2012. Simultaneous measurement of the silicon content and physiological parameters by FTIR spectroscopy in diatoms with siliceous cell walls. Plant Cell Physiol. 53, 2153-2162.

Laurens, L.M.L., Wolfrum, E.J., 2011. Feasibility of spectroscopic characterisation of algal lipids: chemometric correlation of NIR and FTIR spectra with exogenous lipids in algal biomass. Bioenerg. Res. 4, 22-35.

Laurens, L.M.L., Dempster, T.A., Jones, H.D.T., Wolfrum, E.J., Van Wychen, S. McAllister, J.S.P., Rencenberger, M., Parchert, K.J., Gloe, L.M., 2012. Algal biomass constituent analysis: method uncertainties and investigation of the underlying measuring chemistries. Anal. Chem. 84, 1879-1887.

Movasaghi, Z., Rehman, S., Rehmen, I.U., 2008. Fourier Transform Infrared (FTIR) spectroscopy of biological tissues. Appl. Spectrosc. Rev. 43, 134-179.

Murdock, J.H., Wetzel, D.L., 2009. FT-IR microspectroscopy enhances biological and ecological analysis of algae. Appl. Spectrosc. Rev. 44, 335-361.

Nicolai, B.M., Beullens, K., Bobelyn, E., Peirs, A., Saeys, W., Theron, K.I., Lammertyn, J., 2007. Non-destructive measurement of fruit and vegetable quality by means of NIR spectroscopy: a review. Postharvest. Biol. Technol. 46, 99-118.

Nobre, B.P., Villalobos, F., Barragan, B.E., Oliveira, A.C., Batista, A.P., Marques, P.A.S.S., Mendes, R.L., Palavra, A.F., Gouveia, L., 2013. A biorefinery from Nannochloropsis sp. microalga - extraction of oils and pigments. Production of biohydrogen from the leftover biomass. Bioresour. Technol. 135, 128-136.

Palmucci, M., Ratti, S., Giordano, M., 2011. Ecological and evolutionary implication of carbon allocation in marine phytoplankton as a function of nitrogen availability: a Fourier-transform infrared spectroscopy approach. J. Phycol. 47, 313-323.

Pistorius, A.M.A., DeGrip, W.J., Egorova-Zachernyuk, T.A., 2009. Monitoring of biomass composition from microbiological sources by means of FT-IR spectroscopy. Biotechnol. Bioeng. 103, 123-129.

Rodolfi, L., ChiniZittelli, G., Bassi, N., Padovani, G., Biondi, N., Bonini, G., Tredici, M.R. 2009. Microalgae for oil: strain selection, induction of lipid synthesis and outdoor mass cultivation in a low-cost photobioreactor. Biotechnol. Bioeng. $102,100-112$.

Safi, C., Charton, M., Pignolet, O., Silvestre, F., Vaca-Garcia, C., Pontalier, P.-Y., 2013. Influence of microalgae cell wall characteristics on protein extractability and determination of nitrogen-to-protein conversion factors. J. Appl. Phycol. 25, 523-529.

Sills, D.I., Gossett, J.M., 2012. Using FTIR spectroscopy to model alkaline pretreatment and enzymatic saccharification of six lignocellulosic biomasses. Biotechnol. Bioeng. 109, 894-903.

Solovchenko, A., Khozin-goldberg, I., Recht, L., Boussiba, S., 2011. Stress-induced changes in optical properties, pigments and fatty acid content of Nannochloropsis sp.; implications for non-destructive assay of total fatty acids. Mar. Biotechnol. 13, 527-535.

Stehfest, K., Toepel, J., Wilhelm, C., 2005. The application of micro-FTIR spectroscopy to analyse nutrient stress-related changes in biomass composition of phytoplankton algae. Plant Physiol. Biochem. 43, 717-726.

Templeton, D.W., Quinn, M., Van Wychen, S., Hyman, D., Laurens, L.M.L., 2012 Separation and quantification of microalgal carbohydrates. J. Chromatogr. A 1270, 225-234.

Wagner, H., Liu, Z., Langner, U., Stehfest, K., Wilhelm, C., 2010. The use of FTIR spectroscopy to assess quantitative changes in the biochemical composition of microalgae. J. Biophotonics 3, 557-566. 